\title{
Comparación de los patrones de alimentación y defecación de Rhodnius colombiensis y Rhodnius prolixus (Hemiptera, Reduviidae, Triatominae) en condiciones de laboratorio
}

\author{
Andrea Arévalo ${ }^{1}$, Julio César Carranza ${ }^{1}$, Felipe Guhl ${ }^{2}$, Jairo A. Clavijo ${ }^{3}$, \\ Gustavo Adolfo Vallejo ${ }^{1}$ \\ ${ }^{1}$ Laboratorio de Investigaciones en Parasitología Tropical, Facultad de Ciencias, Universidad del Tolima, \\ Ibagué, Colombia. \\ 2 Centro de Investigaciones en Microbiología y Parasitología Tropical, Universidad de los Andes, Bogotá \\ D.C., Colombia. \\ ${ }^{3}$ Departamento de Matemáticas y Estadística, Facultad de Ciencias, Universidad del Tolima, Ibagué, \\ Colombia.
}

Introducción. Rhodnius colombiensis ocasionalmente ingresa a las viviendas humanas por lo cual se ha sugerido que podría desempeñar un importante papel en la transmisión de la tripanosomiasis americana.

Objetivo. Definir el desempeño de $R$. colombiensis como vector, comparando los patrones de alimentación y defecación con los de $R$. prolixus, el principal vector domiciliado de Trypanosoma cruzi en Colombia.

Materiales y métodos. Para cada estadio de $R$. colombiensis y $R$. prolixus se estudió el tiempo promedio para iniciar la picada, el tiempo para alcanzar la repleción, el número de interrupciones y defecaciones durante la comida, el tiempo transcurrido entre el fin de la alimentación y la primera defecación, el número de defecaciones durante 10,60 y 95 min después de la comida y la cantidad de sangre ingerida.

Resultados. El tiempo promedio para iniciar la picada de las ninfas N5, machos y hembras presentaron diferencias significativas entre las dos especies. El promedio de defecaciones por insecto durante los 10 min después de la alimentación fue mayor para cada estadio de $R$. prolixus y presentó diferencias significativas con $R$. colombiensis. Por otro lado, el peso promedio de sangre ingerida por $R$. colombiensis y $R$. prolixus en cada estadio presentó diferencias significativas en N1, N2, N5 y hembras.

Conclusión. $R$. colombiensis presenta menor número de defecaciones que $R$. prolixus durante la comida. Un mayor porcentaje de $R$. prolixus defecan durante los 10,60 y 95 min después de la alimentación. Sin embargo, $R$. colombiensis permanece mayor tiempo asociado al hospedero vertebrado, lo cual aumentaría la probabilidad de transmisión teniendo en cuenta el ingreso ocasional de los adultos a las viviendas humanas y sus elevadas prevalencias con T. cruzi y $T$. rangeli.

Palabras clave: Rhodnius/fisiología, defecación, crecimiento y desarrollo, Trypanosoma, enfermedad de Chagas.

Comparison of feeding and defecation patterns of Rhodnius colombiensis and Rhodnius prolixus (Hemiptera, Reduviidae, Triatominae) under laboratory conditions.

Introduction. Rhodnius colombiensis occasionally comes into human dwellings and consequently its role as an important potential vector in the transmission of American trypanosomiasis has been suggested.

Objective. The potential role of $R$. colombiensis as vector was defined by comparing the feeding and defecation patterns between $R$. colombiensis and $R$. prolixus, the main domiciliary vector of Trypanosoma cruzi in Colombia.

Materials and methods. For each developmental stage of $R$. colombiensis and $R$. prolixus the following data were collected: (1) time of feeding initiation, (2) the time for reaching the repletion, 
(3) the number of interruptions and defecations during the feeding, (4) the time between the end of the feeding and the first defecation, (5) the number of defecations during 10, 60 and 95 minutes of observation after feeding, and (6) the quantity of blood ingested.

Results. The mean time of feeding initiation of the fifth instar nymphs, males and females, showed significant differences between the two species. The average of insects that defecated within 10 minutes after feeding was higher for each successive stage of $R$. prolixus and showed significant differences with Rhodnius colombiensis. In contrast, the mean weight of blood ingested by each stage of $\mathrm{R}$. colombiensis and $R$. prolixus was significantly different between the N1, N2, N5 and females of these species.

Conclusion. Rhodnius colombiensis produced fewer defecations than $R$. prolixus during feeding. A higher percentage of $R$. prolixus defecated within 10,60 and 95 minutes after feeding. However, $R$. colombiensis remains a longer time in contact with the vertebrate host, thus raising the probability of its role in transmission considering its occasional entry to human dwellings and its higher prevalences of infection with $T$. cruzi and $T$. rangeli.

Key words: Rhodnius, physiology, defecation, growth and development, Trypanosoma, Chagas disease.

El género Rhodnius incluye 16 especies, en algunas de las cuales se han descrito las características biológicas, bioquímicas y moleculares (1). R. colombiensis es una especie recientemente descrita, que con $R$. pallescens y R. ecuadoriensis conforman el grupo "pallescens" de la cordillera de los Andes en el noroeste de América del Sur (2). Por otro lado, $R$. colombiensis se ha encontrado asociada a las palmas de vino (Attalea butyraceae) en la cuenca alta del valle del río Magdalena en la región central de Colombia (3), constituyendo un posible riesgo en la transmisión de la enfermedad de Chagas, pues esta especie ocasionalmente ingresa a las viviendas, presentando elevadas prevalencias de $T$. cruzi y $T$. rangeli (Vallejo GA, Lozano LE, Carranza JC, Sánchez JL, Jaramillo JC, Guhl F, et al. Ecología de los triatominos no domiciliados en Colombia con especial referencia a Rhodnius colombiensis en el departamento del Tolima. En: Vallejo GA, Carranza JC y Jaramillo JC editores. Curso Taller Internacional: Biología, Epidemiología y Control de la Tripanosomosis Americana y Leishmaniosis. Ibagué, Colombia 2000; p.1-134).

Los estudios sobre el comportamiento de los triatominos durante la toma del alimento son de

\footnotetext{
Correspondencia:

Gustavo Adolfo Vallejo, Laboratorio de Investigaciones en Parasitología Tropical, Facultad de Ciencias, Universidad del Tolima, A.A. 546, Ibagué, Colombia. Fax (+57-8) 2669176.

gvallejo@ut.edu.co
}

Recibido: 14/03/06; aceptado: 19/05/06 gran importancia para comprender la dinámica de transmisión de $T$. cruzi. Varios autores han demostrado que algunas especies de triatominos no defecan durante, ni tampoco inmediatamente después de la comida, mientras que otras especies son muy eficientes en la transmisión de $T$. cruzi debido a las altas tasas de defecación durante el alimento y durante los primeros minutos después de la alimentación (4). Estas diferencias en los patrones de alimentación y de defecación podrían explicar, al menos en parte, las diferencias en las prevalencias de $T$. cruzien humanos y en reservorios en diferentes regiones de América Latina.

Para contribuir al conocimiento de la biología y el papel de $R$. colombiensis en la transmisión de la enfermedad de Chagas en la región central de Colombia, se estudió para cada estadio de desarrollo el tiempo promedio para iniciar la picada, el tiempo de alimentación hasta la repleción, el número de interrupciones y defecaciones durante la comida, el intervalo entre el fin de la alimentación y la primera defecación, el número de defecaciones durante 10,60 y 95 minutos de observación después de la comida y la cantidad de sangre ingerida. Con fines comparativos, estos mismos parámetros se estudiaron en los diferentes estadios de desarrollo de $R$. prolixus, especie domiciliada que se encuentra en simpatría con $R$. colombiensis en la cuenca alta del río Magdalena.

\section{Materiales y métodos}

$R$. colombiensis fueron capturados en palmas de Attalea butyracea y $R$. prolixus domiciliados 
fueron capturados en la vereda Totarco-Tamarindo en el municipio de Coyaima, departamento del Tolima (Colombia central). Las colonias de ambas especies se mantuvieron a una temperatura de $28 \pm 1^{\circ} \mathrm{C}$, humedad relativa de 75 a $80 \%$ y fotoperiodicidad de 12/12 horas en una incubadora automática B.O.D. Lab-Line. Los insectos fueron alimentados con sangre de gallina semanalmente.

Se compararon las variables sobre alimentación y defecación de $R$. colombiernsis y $R$. prolixus en tres etapas experimentales. En la primera etapa se estudió el comportamiento de los insectos durante el tiempo en que permanecieron tomando el alimento sobre ratones ICR, para lo cual se midió el tiempo promedio de picada, el tiempo promedio para alcanzar la repleción, el promedio de interrupciones por insecto y el promedio de defecaciones durante la comida. En la segunda etapa se estudiaron los patrones de defecación después de la comida, calculándose el tiempo promedio para defecar por primera vez después de la comida y el promedio de defecaciones durante 10, 60 y $95 \mathrm{~min}$. En la tercera etapa se calculó el peso y volumen de sangre ingerida en promedio por cada estadio de $R$. colombiensis y $R$. prolixus y se estimó el número de veces que cada estadio aumentó su peso después de la comida.

\section{Patrones de alimentación y defecación}

Para R. colombiensis se utilizaron 30 individuos de cada estadio, incluidos machos y hembras, para un total de 210 insectos. Para R. prolixus, se utilizaron 203 insectos (30 N1; 30 N2; 30 N3; $30 \mathrm{~N} 4 ; 30 \mathrm{~N} 5 ; 30$ machos y 23 hembras). Cada individuo fue identificado con marcas de tinta blanca en el dorso para facilitar la observación.

Grupos de tres a seis insectos fueron alimentados hasta la repleción sobre ratones ICR sedados con maleato de acepromacina e inmovilizados con una malla de nylon. Cada ratón fue puesto en un recipiente transparente y sujeto con cinta de enmascarar para evitar movimientos bruscos. Los insectos se dejaron en el contenedor entre $60 \mathrm{y}$ 180 minutos en un cuarto ligeramente oscuro con una temperatura promedio de $28^{\circ} \mathrm{C}$ y un mismo observador los monitorizó continuamente sin mover el recipiente durante el experimento. Los tiempos para iniciar la picada y para alcanzar la repleción se cronometró, se calculó el promedio de defecaciones durante la comida, así mismo, se contaron las interrupciones y las defecaciones durante y después de la comida.

\section{Cantidad de sangre ingerida}

En un experimento independiente se calculó la cantidad de sangre ingerida, para lo cual se utilizaron $210 R$. colombiensis y $210 R$. prolixus (30 por cada estadio de desarrollo). Los insectos fueron sometidos a 15 días de ayuno, pesados antes y después de la alimentación en una balanza analítica y alimentados con sangre de gallina. Cada individuo se colocó en un vial previamente marcado para llevar el correspondiente registro.

\section{Análisis estadístico}

Para cada variable se calcularon las medias con los respectivos intervalos de $95 \%$ de confianza. Se utilizó análisis multivariado de varianza (MANOVA) para comparar los promedios de las variables en cada estadio de desarrollo de las dos especies. Para los patrones de alimentación, de defecación y la cantidad de sangre ingerida de las ninfas se usó un diseño factorial $5 \times 2$ balanceado con 30 réplicas. Para los patrones de alimentación y defecación de los adultos, el diseño factorial fue $2 \times 2$ desbalanceado. Para la cantidad de sangre ingerida por los adultos se utilizó un diseño factorial $2 \times 2$ balanceado. Para las comparaciones se realizó la prueba de Duncan. El software utilizado fue SAS versión 8.2.

\section{Resultados}

\section{Patrones de alimentación y defecación durante la comida}

En el cuadro 1 se presentan los resultados del comportamiento de $R$. colombiensis y $R$. prolixus durante la comida. Se observó que los tiempos promedios antes de iniciar la picada (tiempo de ataque), no presentaron diferencias significativas entre las dos especies cuando se compararon las $\mathrm{N} 1$, N2, N3 y N4; sin embargo, el tiempo promedio de ataque de N5, machos y hembras, en $R$. colombiensis fue mayor que en $R$. prolixus, con diferencias significativas entre las dos especies.

El tiempo promedio para alcanzar la repleción fue mayor en N2 y N5 $R$. colombiensis, con 
Cuadro 1. Comparación del tiempo para iniciar la comida (tiempo de ataque), tiempo de comida hasta repleción, interrupciones y defecaciones durante la alimentación de Rhodnius colombiensis y Rhodnius prolixus sobre ratón Mus musculus. El tiempo está expresado en minutos:segundos.

\begin{tabular}{|c|c|c|c|c|c|c|c|c|c|c|c|c|}
\hline \multirow[t]{2}{*}{ Estadio } & \multicolumn{3}{|c|}{$\begin{array}{c}\text { Tiempo de ataque } \\
\text { (intervalo de confianza del 95\%) }\end{array}$} & \multicolumn{3}{|c|}{$\begin{array}{l}\text { Tiempo para comer hasta repleción } \\
\text { (intervalo de confianza del } 95 \% \text { ) }\end{array}$} & \multicolumn{3}{|c|}{$\begin{array}{l}\text { Interrupciones por insecto } \\
\text { (intervalo de confianza del 95\%) }\end{array}$} & \multicolumn{3}{|c|}{$\begin{array}{l}\text { Defecaciones durante la comida } \\
\text { (intervalo de confianza del } 95 \% \text { ) }\end{array}$} \\
\hline & $\begin{array}{l}\text { Rhodnius } \\
\text { colombiensis }\end{array}$ & $\begin{array}{l}\text { Rhodnius } \\
\text { prolixus }\end{array}$ & $\begin{array}{c}\text { Valores } \\
p\end{array}$ & $\begin{array}{c}\text { Rhodnius } \\
\text { colombiensis }\end{array}$ & $\begin{array}{l}\text { Rhodnius } \\
\text { prolixus }\end{array}$ & $\begin{array}{l}\text { Valores } \\
\qquad p\end{array}$ & $\begin{array}{l}\text { Rhodnius } \\
\text { colombiensis }\end{array}$ & $\begin{array}{l}\text { Rhodnius } \\
\text { prolixus }\end{array}$ & $\begin{array}{l}\text { Valores } \\
\quad p\end{array}$ & $\begin{array}{c}\text { Rhodnius } \\
\text { colombiensis }\end{array}$ & $\begin{array}{l}\text { Rhodnius } \\
\text { prolixus }\end{array}$ & $\begin{array}{l}\text { Valores } \\
p\end{array}$ \\
\hline N1 & $\begin{array}{c}0: 10,5 \\
\left(0^{*}-0: 27,7\right)\end{array}$ & $\begin{array}{c}0: 7 \\
(0: 2,8-0: 11,2)\end{array}$ & 0,6867 & $\begin{array}{c}13: 3 \\
(10: 6,3-15: 59,7)\end{array}$ & $\begin{array}{c}12: 31,8 \\
(10: 0,9-15: 2,7)\end{array}$ & 0,7851 & 0 & $0,3(0,02-0,6)$ & 0,0324 & $\begin{array}{c}0,07 \\
(0-0,2)\end{array}$ & $\begin{array}{c}0,3 \\
(0,03-0,5)\end{array}$ & 0,1169 \\
\hline N2 & $\begin{array}{c}0: 7,1 \\
(0: 0,5-0: 13,7)\end{array}$ & $\begin{array}{c}0: 1,7 \\
(0: 1,1-0: 2,4)\end{array}$ & 0,1025 & $\begin{array}{c}13: 57 \\
(10: 39,4-17: 14,5)\end{array}$ & $\begin{array}{c}10: 11,2 \\
(9: 1,8-11: 20,5)\end{array}$ & 0,0315 & $0,3\left(0^{*}-0,7\right)$ & $0,3(0,02-0,6)$ & 0,9018 & 0 & $\begin{array}{c}0,2 \\
(0,04-0,4)\end{array}$ & 0,0139 \\
\hline N3 & $\begin{array}{c}0: 4,4 \\
(0: 1,4-0: 7,4)\end{array}$ & $\begin{array}{c}0: 4,9 \\
(0: 1,8-0: 8,1)\end{array}$ & 0,8144 & $\begin{array}{c}13: 56,4 \\
(11: 31,6-16: 21,2)\end{array}$ & $\begin{array}{c}14: 51 \\
(12: 29-17: 13,3)\end{array}$ & 0,5854 & $0,2\left(0^{*}-0,4\right)$ & $0,8(0,4-1,3)$ & 0,0145 & 0 & $\begin{array}{c}0,4 \\
(0,2-0,6)\end{array}$ & 0,0006 \\
\hline N4 & $\begin{array}{c}1: 8,1 \\
(0: 25,2-1: 50,9)\end{array}$ & $\begin{array}{c}0: 23 \\
(0: 4,1-0: 41,9)\end{array}$ & 0,0538 & $\begin{array}{c}22: 12 \\
(18: 8-26: 16)\end{array}$ & $\begin{array}{c}18: 44,4 \\
(15: 21-22: 7,8)\end{array}$ & 0,1866 & $0,3(0,05-0,5)$ & $0,5(0,2-0,9)$ & 0,2148 & 0 & $\begin{array}{c}0,8 \\
(0,1-1,4)\end{array}$ & 0,0214 \\
\hline N5 & $\begin{array}{c}3: 33,4 \\
(2: 13,1-4: 53,8)\end{array}$ & $\begin{array}{c}1: 39 \\
(0: 35,1-2: 42,1)\end{array}$ & 0,0255 & $\begin{array}{c}30: 48 \\
(25: 35-36: 1)\end{array}$ & $\begin{array}{c}23: 13,3 \\
(18: 48,3-27: 38,3)\end{array}$ & 0,0271 & $1,5(0,4-2,6)$ & $0,9(0,4-1,5)$ & 0,4045 & 0 & $\begin{array}{c}0,4 \\
(0,04-0,8)\end{array}$ & 0,0274 \\
\hline Hembras & $\begin{array}{c}1: 56 \\
(0: 49,1-3: 2,8)\end{array}$ & $\begin{array}{c}0: 14,9 \\
\left(0^{*}-0: 38,6\right)\end{array}$ & 0,0118 & $\begin{array}{c}20: 21,3 \\
(14: 21,1-26: 21,4)\end{array}$ & $\begin{array}{c}19: 13 \\
(13: 20,1-25: 5)\end{array}$ & 0,7847 & $1,5(0,8-2,1)$ & $1,5(0,9-2,1)$ & 0.9785 & 0 & $\begin{array}{c}0,3 \\
(0-0,8)\end{array}$ & 0,1034 \\
\hline Machos & $\begin{array}{c}1: 48 \\
(0: 7,1-3: 29)\end{array}$ & $\begin{array}{c}0: 8,2 \\
(0: 0,05-0: 16,3)\end{array}$ & 0,0484 & $\begin{array}{c}19: 23,1 \\
(13: 30,3-25: 16)\end{array}$ & $\begin{array}{c}13: 36 \\
(10: 51-16: 21,2)\end{array}$ & 0,0736 & $1,1(0,3-1,9)$ & $0,7(0,4-0,9)$ & 0,3032 & $\begin{array}{c}0,03 \\
(0-0,1)\end{array}$ & 0 & 0,3215 \\
\hline
\end{tabular}

diferencias significativas entre las dos especies (cuadro 1).

De acuerdo a las observaciones realizadas, la mayoría de los estadios de desarrollo de $R$. colombiensis y $R$. prolixus interrumpieron la toma del alimento; la única excepción fue $\mathrm{N} 1$ de $R$. colombiensis, pues ningún insecto de este estadio interrumpió su alimentación. El promedio de interrupciones por insecto entre $R$. colombiensis y $R$. prolixus presentó diferencias significativas en N1 y N3.

Por otro lado, el promedio de defecaciones por insecto durante la comida fue mayor en $R$. prolixus, presentando diferencias significativas con los mismos estadios de $R$. colombiensis; los $\mathrm{N} 1$, las hembras y los machos no presentaron diferencias significativas al comparar el promedio de defecaciones durante la comida en las dos especies.

\section{Patrones de defecación después de la comida}

El cuadro 2 presenta los patrones de defecación de $R$. colombiensis y $R$. prolixus después de la toma del alimento. El tiempo promedio para defecar por primera vez después de la toma del alimento fue mayor en todos los estadios de $R$. colombiensis, presentando diferencias significativas con los estadios de $R$. prolixus. Por otro lado, el promedio de defecaciones por insecto durante los primeros 10 minutos fue mayor en todos los estadios de $R$. prolixus, presentando diferencias significativas con los estadios de $R$. colombiensis. Así mismo, el promedio de defecaciones acumuladas por insecto durante 60 minutos de observación fue mayor en $R$. prolixus, presentando diferencias significativas con las $\mathrm{N} 1$, N3, N4, N5 y hembras de $R$. colombiensis. De igual manera, después de 95 minutos de observación, el promedio de defecaciones acumuladas por insecto fue mayor en $R$. prolixus, presentando diferencias significativas con N1, N3, $\mathrm{N} 4$, N5, hembras y machos de $R$. colombiensis (cuadro 2).

\section{Cantidad de sangre ingerida}

La comparación de la sangre ingerida por los distintos estadios de $R$. colombiensis y de $R$. prolixus se muestra en el cuadro 3 . El peso promedio y el volumen promedio de sangre ingerida presentó diferencias significativas entre N1, N2, N5 y hembras de las dos especies; las ninfas N3, $\mathrm{N} 4$ y los machos no presentaron diferencias significativas en el peso y volumen de sangre ingerida. Con relación al número de veces que cada estadio aumentó su peso inicial después de la comida, se observaron diferencias significativas entre las N2, N5 y hembras; los demás estadios no presentaron diferencias significativas. Las N5 
Cuadro 2. Comparación del tiempo para defecar por primera vez después de la comida; defecaciones después de diez minutos, una hora y noventa y cinco minutos entre Rhodnius colombiensis y $R$. prolixus. El tiempo está expresado en minutos:segundos. Las diferencias fueron significativas cuando $p<0,05$.

\begin{tabular}{|c|c|c|c|c|c|c|c|c|c|c|c|c|}
\hline \multirow[b]{2}{*}{ Estadio } & \multicolumn{3}{|c|}{$\begin{array}{l}\text { Tiempo para defecar por primera vez } \\
\text { después de la comida. } \\
\text { (intervalo de confianza del 95\%) }\end{array}$} & \multicolumn{3}{|c|}{$\begin{array}{l}\text { Defecaciones por insecto durante } \\
\text { los primeros } 10 \text { minutos } \\
\text { (intervalo de confianza del 95\%) }\end{array}$} & \multicolumn{3}{|c|}{$\begin{array}{l}\text { Defecaciones por insecto durante } \\
\text { la primera hora } \\
\text { (intervalo de confianza del 95\%) }\end{array}$} & \multicolumn{3}{|c|}{$\begin{array}{l}\text { Defecaciones por insecto durante } \\
\text { durante los } 95 \text { minutos } \\
\text { (intervalo de confianza del } 95 \% \text { ) }\end{array}$} \\
\hline & $\begin{array}{c}\text { Rhodnius } \\
\text { colombiensis }\end{array}$ & $\begin{array}{l}\text { Rhodnius } \\
\text { prolixus }\end{array}$ & Valores $p$ & $\begin{array}{l}\text { Rhodnius } \\
\text { colombiensis }\end{array}$ & $\begin{array}{l}\text { Rhodnius } \\
\text { prolixus }\end{array}$ & Valores $p$ & $\begin{array}{c}\text { Rhodnius } \\
\text { colombiensis }\end{array}$ & $\begin{array}{l}\text { Rhodnius } \\
\text { s prolixus }\end{array}$ & Valores $p$ & $\begin{array}{c}\text { Rhodnius } \\
\text { colombiensis }\end{array}$ & $\begin{array}{l}\text { Rhodnius } \\
\text { s prolixus }\end{array}$ & s Valores $p$ \\
\hline N1 & $\begin{array}{c}38: 12,6 \\
(29: 23-47: 2,6)\end{array}$ & $\begin{array}{c}11: 10,8 \\
(7: 4,2-15: 17,4)\end{array}$ & 0,0001 & $\begin{array}{c}0,07 \\
(0-0,2)\end{array}$ & $\begin{array}{c}0,8 \\
(0,5-1,0)\end{array}$ & 0,0001 & $\begin{array}{c}2,3 \\
(1,6-2,9)\end{array}$ & $\begin{array}{c}3,7 \\
(2,8-4,4)\end{array}$ & 0,0109 & $\begin{array}{c}3,7 \\
(2,8-4,5)\end{array}$ & $\begin{array}{c}5,5 \\
(4,6-6,5)\end{array}$ & 0,0044 \\
\hline N2 & $\begin{array}{c}30: 32,4 \\
(23: 0,8-38: 3,9)\end{array}$ & $\begin{array}{c}11: 37,2 \\
(5: 54,9-17: 19,5)\end{array}$ & 0,0001 & $\begin{array}{c}0,07 \\
(0-0,2)\end{array}$ & $\begin{array}{c}0,9 \\
(0,6-1,1)\end{array}$ & 0,0001 & $\begin{array}{c}2,3 \\
(1,8-2,9)\end{array}$ & $\begin{array}{c}3,2 \\
(2,3-4)\end{array}$ & 0,0897 & $\begin{array}{c}4,2 \\
(3,4-5,1)\end{array}$ & $\begin{array}{c}4,8 \\
(3,7-5,8)\end{array}$ & 0,4329 \\
\hline N3 & $\begin{array}{c}33: 48 \\
(26: 16,5-41: 20)\end{array}$ & $\begin{array}{c}11: 46 \\
(6: 22,9-17: 8,3)\end{array}$ & 0,0001 & $\begin{array}{c}0,1 \\
(0-0,2)\end{array}$ & $\begin{array}{c}0,9 \\
(0,2-0,6)\end{array}$ & ${ }^{1} 0.0001$ & $\begin{array}{c}2,1 \\
(1,5-2,6)\end{array}$ & $\begin{array}{c}3,8 \\
(3,6-4,5)\end{array}$ & 0,0001 & $\begin{array}{c}3,8 \\
(3,1-4,4)\end{array}$ & $\begin{array}{c}5,7 \\
(4,9-6,6)\end{array}$ & 0,0004 \\
\hline N4 & $\begin{array}{c}54: 46 \\
(43: 13,4-66: 18,2)\end{array}$ & $\begin{array}{c}9: 54,8 \\
(3: 7-16: 42,5)\end{array}$ & 0.0001 & $\begin{array}{c}0,03 \\
(0-0,1)\end{array}$ & $\begin{array}{c}0,8 \\
(0,6-1,1)\end{array}$ & 0,0001 & $\begin{array}{c}1 \\
0,6-1,4)\end{array}$ & $\begin{array}{c}3,0 \\
(2,4-3,5)\end{array}$ & 0,0001 & $\begin{array}{c}2,1 \\
(1,4-2,7)\end{array}$ & $\begin{array}{c}4,1 \\
(3,4-4,8)\end{array}$ & 0,0001 \\
\hline N5 & $\begin{array}{c}53: 43,5 \\
(42: 0,7-65: 26,2)\end{array}$ & $\begin{array}{c}12: 3,6 \\
(4: 58,6-19: 8,7)\end{array}$ & 0.0001 & $\begin{array}{c}0,07 \\
(0-0,2)\end{array}$ & $\begin{array}{c}0,8 \\
(0,5-1,1)\end{array}$ & 0,0001 & $\begin{array}{c}0,7 \\
(0,4-1)\end{array}$ & $\begin{array}{c}4,6 \\
(3,6-5,7)\end{array}$ & 0,0001 & $\begin{array}{c}1,7 \\
(1,2-2,2)\end{array}$ & $\begin{array}{c}6,4 \\
(5,0-7,7)\end{array}$ & 0,0001 \\
\hline Hembras & $\begin{array}{c}58: 27,1 \\
(48: 22,1-68: 32)\end{array}$ & $\begin{array}{c}22: 22 \\
(7: 7-37: 37)\end{array}$ & 0,0001 & $\begin{array}{c}0,07 \\
(0-0,2)\end{array}$ & $\begin{array}{c}0,9 \\
(0,6-1,2)\end{array}$ & 0.0001 & $\begin{array}{c}0,7 \\
(0,3-0,9)\end{array}$ & $\begin{array}{c}3,7 \\
(2,5-5)\end{array}$ & 0,0001 & $\begin{array}{c}1,5 \\
(1,0-2,0)\end{array}$ & $\begin{array}{c}5,5 \\
(3,9-7,0)\end{array}$ & 0,0001 \\
\hline Machos & $\begin{array}{c}68: 28,2 \\
(57: 24,4-79: 32)\end{array}$ & $\begin{array}{c}47: 34 \\
(33: 32-61: 35)\end{array}$ & 0,0199 & $\begin{array}{c}0,03 \\
(0-0,1)\end{array}$ & $\begin{array}{c}0,4 \\
(0,1-0,6)\end{array}$ & 0,0108 & $\begin{array}{c}0,7 \\
(0,3-1,1)\end{array}$ & $\begin{array}{c}1,5 \\
(0,8-2,1)\end{array}$ & 0,0501 & $\begin{array}{c}1,3 \\
(0,7-1,9)\end{array}$ & $\begin{array}{c}2,6 \\
(1,7-3,5)\end{array}$ & 0,0240 \\
\hline
\end{tabular}

Cuadro 3. Comparación de la cantidad de sangre ingerida por cada estadio de desarrollo de Rhodnius colombiensis y Rhodnius prolixus. El nivel de significación es de 0,05. Las diferencias fueron significativas cuando $p<0,05$.

\begin{tabular}{|c|c|c|c|c|c|c|c|c|c|}
\hline \multirow[t]{2}{*}{$\begin{array}{l}\text { Estadios } \\
\text { Variables }\end{array}$} & \multicolumn{3}{|c|}{$\begin{array}{l}\text { Promedio de sangre ingerida (mg) } \\
\text { (Intervalo de confianza del } 95 \% \text { ) }\end{array}$} & \multicolumn{3}{|c|}{$\begin{array}{l}\text { Volumen de sangre ingerida }(\mu \mathrm{l}) \\
\text { (Intervalo de confianza del } 95 \% \text { ) }\end{array}$} & \multicolumn{3}{|c|}{$\begin{array}{l}\text { Número de veces que aumenta su } \\
\text { peso inicial después de la comida } \\
\text { (Intervalo de confianza del } 95 \%\end{array}$} \\
\hline & $\begin{array}{c}\text { Rhodnius } \\
\text { colombiensis }\end{array}$ & $\begin{array}{l}\text { Rhodnius } \\
\text { prolixus }\end{array}$ & Valores $\mathrm{p}$ & $\begin{array}{l}\text { Rhodnius } \\
\text { colombiensis }\end{array}$ & $\begin{array}{c}\text { Rhodnius } \\
\text { prolixus }\end{array}$ & Valores $p$ & $\begin{array}{c}\text { Rhodnius } \\
\text { colombiensis }\end{array}$ & $\begin{array}{c}\text { Rhodnius } \\
\text { prolixus }\end{array}$ & Valores $p$ \\
\hline $\mathrm{N} 1$ & $\begin{array}{c}3,3 \\
(3,0-3,5)\end{array}$ & $\begin{array}{c}2,9 \\
(2,9-3,1)\end{array}$ & 0,0339 & $\begin{array}{c}3,1 \\
(2,8-3,3)\end{array}$ & $\begin{array}{c}2,8 \\
(2,7-2,9)\end{array}$ & 0,0338 & $\begin{array}{c}7,7 \\
(6,6-8,7)\end{array}$ & $\begin{array}{c}7,7 \\
(6,9-8,4)\end{array}$ & 0,9842 \\
\hline N2 & $\begin{array}{c}9,4 \\
(8,6-10,2)\end{array}$ & $\begin{array}{c}10,6 \\
(9,7-11,5)\end{array}$ & 0,0445 & $\begin{array}{c}8,7 \\
(7,7-9,6)\end{array}$ & $\begin{array}{c}10,1 \\
(9,3-11)\end{array}$ & 0,0205 & $\begin{array}{c}7 \\
(6,4-7,7)\end{array}$ & $\begin{array}{c}8,9 \\
(8,0-9,7)\end{array}$ & 0.0009 \\
\hline N3 & $\begin{array}{c}27,8 \\
(24,8-30,8)\end{array}$ & $\begin{array}{c}30,1 \\
(27,3-32,9)\end{array}$ & 0,2425 & $\begin{array}{c}26,7 \\
(23,8-29,5)\end{array}$ & $\begin{array}{c}28,9 \\
(26,3-31,6)\end{array}$ & 0,2387 & $\begin{array}{c}8,8 \\
(7,8-9,7)\end{array}$ & $\begin{array}{c}8,4 \\
(7,7-9,0)\end{array}$ & 0,4424 \\
\hline N4 & $\begin{array}{c}74,3 \\
(64,4-84,2)\end{array}$ & $\begin{array}{c}77,6 \\
(70,8-84,4)\end{array}$ & 0,5789 & $\begin{array}{c}71,5 \\
(62-81)\end{array}$ & $\begin{array}{c}74,6 \\
(68,1-81,2)\end{array}$ & 0,58 & $\begin{array}{c}8,5 \\
(7,4-9,6)\end{array}$ & $\begin{array}{c}8,2 \\
(7,3-9,0)\end{array}$ & 0,6386 \\
\hline N5 & $\begin{array}{c}237,9 \\
(215,7-260,2)\end{array}$ & $\begin{array}{c}207 \\
(188,4-225,6)\end{array}$ & 0,0332 & $\begin{array}{c}228,9 \\
(207,6-250,4)\end{array}$ & $\begin{array}{c}199,2 \\
181,3-217,1)\end{array}$ & 0,0332 & $\begin{array}{c}8,6 \\
(7,6-9,6)\end{array}$ & $\begin{array}{c}7,4 \\
(6,7-8,1)\end{array}$ & 0,0444 \\
\hline Hembras & $\begin{array}{c}99,6 \\
(82,6-116,6)\end{array}$ & $\begin{array}{c}122,8 \\
(110,4-135,2)\end{array}$ & 0,0281 & $\begin{array}{c}95,8 \\
(79,4-112,2)\end{array}$ & $\begin{array}{c}118,2 \\
06,2-130,1)\end{array}$ & 0,0280 & $\begin{array}{c}2,2 \\
(1,9-2,6)\end{array}$ & $\begin{array}{c}2,9 \\
(2,6-3,2)\end{array}$ & 0,0054 \\
\hline Machos & $\begin{array}{c}65,4 \\
(57,6-73,3)\end{array}$ & $\begin{array}{c}69,4 \\
(60-78,7)\end{array}$ & 0,5137 & $\begin{array}{c}62,9 \\
(55,4-70,5)\end{array}$ & $\begin{array}{c}66,7 \\
(57,7-75,7)\end{array}$ & 0,5147 & $\begin{array}{c}2 \\
(1,9-2,2)\end{array}$ & $\begin{array}{c}2,2 \\
(2-2,5)\end{array}$ & 0,1249 \\
\hline
\end{tabular}

de las dos especies fueron los estadios con mayor avidez por el alimento, pues ingirieron entre dos y tres veces más sangre que el promedio de los adultos. En los adultos de ambas especies, los machos ingirieron menos sangre que las hembras (cuadro 3).

\section{Discusión}

\section{Patrones de alimentación y defecación durante} la comida

La comparación entre $R$. colombiensis y $R$. prolixus mostró evidentes diferencias en los 
patrones de alimentación y defecación entre las dos especies y entre los diferentes estadios dentro de cada especie. Una interesante observación fue que las ninfas N1, N2, N3 y N4 de $R$. colombiensis y $R$. prolixus no presentaron diferencias significativas en el tiempo promedio para iniciar la picada sobre ratones ICR, mostrando el mismo grado de avidez por la toma del alimento en las condiciones experimentales utilizadas. Por otro lado, las N5, los machos y las hembras de $R$. prolixus iniciaron más rápido la toma del alimento que los mismos estadios de $R$. colombiensis (cuadro 1). Aunque los adultos de R. colombiensis son más tímidos para la toma del alimento, ingresan a las viviendas atraídos por la luz o por diferentes fuentes de alimento. La frecuencia con que los adultos de $R$. colombiensis ingresan a las viviendas fue confirmada en la encuesta entomológica realizada en el departamento del Tolima en el año 2000, en la cual se capturaron adultos de $R$. colombiensis en el interior de las viviendas de los municipios de Alvarado, Carmen de Apicalá, Coyaima, Coello, Guamo, Ibagué, Icononzo, Lérida, Libano, Ortega, Prado, Purificación, Santa Isabel y San Luis, los cuales constituían el 33\% de los municipios encuestados (Guhl F et al. Implementación de la Etapa III del Programa Nacional de Prevención y Control de la Enfermedad de Chagas y la Cardiopatía Infantil. Región Centro Oriental: Departamento del Tolima. Informe Final. Universidad de los Andes). Previas observaciones en el municipio de Coyaima, Tolima, mostraron que en $R$. colombiensis adultos capturados en el interior de las viviendas y en adultos capturados en las palmas aledañas a las viviendas, la infección por T. cruzi alcanzó una prevalencia del $98 \%$ (datos no publicados). Por otro lado, el examen de $396 R$. prolixus domiciliados en la misma área geográfica mostró una prevalencia general del 3,03\% de T. cruzi (5).

De conformidad con lo anterior se podría esperar que cualquier adulto de $R$. colombiensis que logre picar y defecar sobre un vertebrado del domicilio humano presentaría mayor probabilidad para la transmisión de $T$. cruzique las ninfas y los adultos de $R$. prolixus domiciliados en esta región.

La comparación de los datos obtenidos en el presente estudio con datos de otros autores es difícil debido a las diferencias en variables experimentales como la temperatura ambiente, humedad relativa y fuente de alimento, o al uso de pequeños números de insectos, entre otras. Adicionalmente, existen variaciones inter e intraespecíficas en el comportamiento de triatominos como Triatoma dimidiata, en la que se observó que las ninfas de primer estadio son más tímidas para iniciar la picada que los restantes estadios (4). Por otro lado, Rocha da Silva D. et al mostraron que los adultos de $R$. pictipes presentan mayor timidez y que, por lo tanto, en ellos transcurre mayor tiempo entre el ofrecimiento de la comida y la picada (6). Igualmente se observó en los adultos de $T$. brasilensis un mayor tiempo entre el ofrecimiento de la comida y la picada que en los demás estados ninfales; en T. pseudomaculata las N4 fueron más tímidas para la toma del alimento que los demás estadios ninfales (7). Una posible causa de esta variabilidad en el comportamiento de los triatominos con relación al tiempo transcurrido entre el ofrecimiento de la comida y la picada podría ser la fuente de alimento, como fue sugerido por Martínez-lbarra et al., quienes observaron que cuando Meccus picturatus es alimentado sobre gallinas, los adultos son más tímidos para iniciar la picada que los restantes estadios ninfales, pero cuando esta misma especie es alimentada sobre conejos, las N4 son las más tímidas (8).

El tiempo desde la picada hasta la repleción total del insecto fue mayor en $R$. colombiensis, que presentó diferencias significativas con las N2 y N5 de $R$. prolixus; en los demás estadios de desarrollo no se presentaron diferencias significativas. En el presente trabajo se destaca el hecho de que las N5 de R. colombiensis y de $R$. prolixus presentaron el mayor tiempo para obtener la repleción; otros autores también han reportado que las $\mathrm{N} 5$ de diferentes especies gastan mayor tiempo para la repleción, como fue observado en N5 de $T$. dimidata y $R$. prolixus (4), N5 de $T$. brasiliensis y $T$. seudomaculata (7) y N5 de $M$. picturatus (8). Una explicación a este especial comportamiento de las N5 estaría relacionada con que precisamente las N5 son los estadios que mayor cantidad de sangre ingieren con relación a todos los demás estadios de 
desarrollo, lo que a su vez demanda mayor tiempo para alcanzar la repleción, pues las N5 requieren una suficiente cantidad de alimento para la adquisición de nuevas estructuras anatómicas durante la muda al estado adulto. Los tiempos para alcanzar la repleción disminuyeron en los adultos de ambos sexos de $R$. colombiensis y $R$. prolixus; de igual manera, se observó disminución en los adultos de $T$. dimidiata, $T$. infestans y $R$. prolixus (4), T. brasiliensisy T. pseudomaculata (7).

Trabajos previos también mostraron una extensa variación, puesto que el tiempo hasta la repleción se ve afectado por el tipo de alimento y por el tamaño de la especie del insecto, entre otras variables. En este orden de ideas, Zeledón et al. (4) mostraron que los promedios generales del tiempo para alcanzar la repleción son mayores en $T$. dimidiata y $T$. infestans que en $R$. prolixus.

Algunos autores plantean una relación directa entre los patrones de defecación antes de finalizar la comida y las frecuentes interrupciones durante ésta, como sucede con T. dimidiata (4). Por otro lado, se pueden presentar variaciones en las interrupciones durante el proceso de alimentación, incluso entre las mismas especies, posiblemente por el origen de la cepa o por la temperatura bajo la cual se realizan las observaciones $(4,9)$. Para explicar la razón de las interrupciones se han observado las señales eléctricas de la bomba cibarial de varias especies de Rhodnius cuando ésta se contrae para succionar la sangre. Se concluyó que las interrupciones probablemente se deben al hallazgo de otro vaso sanguíneo por parte del vector, ya que en muchas ocasiones el proceso de alimentación fue interrumpido aun sin retirar el rostro del huésped, lo cual se verificó por señales eléctricas irregulares en la bomba cibarial (Viana Sant' Anna MR, Diotaiuti L, Figueiredo Gontijo A, Figueiredo Gontijo N, Pereira MH. Feeding behaviour of morphologically similar Rhodnius species (Hemiptera: Reduviidae): influence of mechanical characteristics and salivary function. En: Guhl F, Schofield CJ, editors. Cuarto Taller Internacional sobre Genética Poblacional y Control de Triatominos (ECLAT 4). Punta Iguana, Cartagena, Colombia, 2002. p. 139-50).

Los únicos estadios de $R$. colombiensis que defecaron durante la comida fueron las $\mathrm{N} 1$ y los machos. Así mismo, el mayor promedio de interrupciones durante la comida lo exhibieron las N5 y las hembras, pero en estos estadios no se llevó a cabo ninguna defecación durante la toma del alimento (cuadro 1). El patrón descrito mostró que no existe una relación directa entre el número de interrupciones y el número de defecaciones durante la comida en $R$. colombiensis, es decir que existieron individuos que defecaron durante la alimentación sin interrumpir tal proceso, o que lo interrumpieron sin efectuar ninguna defecación durante éste. En $R$. prolixus se observó que el promedio de defecaciones durante la toma del alimento fue mayor y presentó diferencias significativas con N2, N3, N4 y N5 de $R$. cololombiensis. Por otro lado, en todos los estadios de $R$. prolixus se presentaron interrupciones durante la comida, indicando una posible relación entre las interrupciones y las defecaciones durante la comida. Esta diferencia entre $R$. prolixus y $R$. colombiensis en relación con el número de defecaciones durante la toma del alimento indica que durante la toma del alimento $R$. prolixus tiene mayor probabilidad de transmisión de $T$. cruzi que $R$. colombiensis.

\section{Patrones de defecación después de la comida}

Como se observó en el cuadro 2, el promedio de defecaciones por insecto durante los primeros 10 , 60 y 95 minutos después de la comida fue mayor en $R$. prolixus y presentó diferencias significativas con $R$. colombiensis. Por otro lado, al igual que en otros estudios, se observó que los patrones más bajos de defecación los presentaron los machos en las dos especies estudiadas (4,10-12).

Sin embargo, el comportamiento de las dos especies después de la toma del alimento puede afectar la eficiencia de la transmisión de $T$. cruzi. Se observó que independientemente del estadio, cuando $R$. prolixus finaliza la toma del alimento huye velozmente, pues está adaptado para refugiarse en grietas o en estructuras anexas al domicilio, de manera que las defecaciones después de la toma del alimento son depositadas lejos del huésped vertebrado, principalmente en paredes y techos de palma. Por el contrario, cuando $R$. colombiensis finaliza la toma del alimento, permanece junto al huésped. Este 
comportamiento podría explicarse porque en condiciones naturales vive estrechamente asociado a nidos de Didelphis marsupialis, en espacios muy reducidos conformados por las brácteas de las palmas de vino $A$. butyraceae, y permanece y defeca muy cerca o sobre el huésped vertebrado. De esta manera, $R$. colombiensis compensaría la falta de defecación durante el alimento, asegurando una eficiente transmisión para $D$. marsupialis, en la que se han observado prevalencias del $89 \%$ de $T$. cruzi en individuos capturados en Coyaima (Tolima) (datos no publicados).

Como se mencionó anteriormente, el promedio de defecaciones durante la toma del alimento promueve la transmisión de $T$. cruzi directamente sobre el vertebrado por parte de $R$. prolixus, pero en menor grado por $R$. colombiensis. Sin embargo, aunque el promedio de defecaciones después de 10,60 y 95 minutos es mayor en $R$. prolixus que en $R$. colombiensis, las diferencias en el comportamiento entre las dos especies podrían afectar la eficiencia de la transmisión, pues como se mencionó, $R$. prolixus huye del huésped y $R$. colombiensis permanece cerca de él, de manera que después de la toma del alimento compensaría su eficiencia para la transmisión del parásito. Aunque no existen datos sobre la relación de $R$. colombiensis y los vertebrados domésticos, asumimos que cuando adultos de $R$. colombiensis ingresan esporádicamente al interior de las viviendas humanas, podrían permanecer cerca de los vertebrados, aumentando así la probabilidad de transmisión de $T$. cruzi.

\section{Cantidad de sangre ingerida}

La cantidad y calidad del alimento ingerido es importante en el desarrollo de los triatominos debido a que el número de huevos producidos por las hembras está directamente relacionado con la cantidad de sangre ingerida (13-16). El peso promedio de la cantidad de sangre ingerida varía de una especie a otra, llegando a ser diferente en muchos casos dentro de la misma especie, inclusive cuando se utiliza la misma fuente de alimento (13). Las N5 necesitan obtener suficientes reservas energéticas para efectuar la última muda hacia el estadio adulto; por tal razón, los promedios de la cantidad de sangre ingerida llegaron a duplicar la cantidad ingerida por las hembras y a triplicar la ingerida por los machos.

La cantidad de sangre que ingiere $R$. colombiensis es similar a la que ingieren otras especies selváticas como $R$. pictipes alimentado artificialmente y sobre ratón (13) y $R$. brethesi (17).

Cuando se comparan los patrones de alimentación y defecación de $R$. colombiensis con los de $R$. prolixus, se observa que esta última especie presenta un comportamiento que favorece la transmisión de $T$. cruzi, por cuanto $R$. prolixus presenta mayor número de defecaciones durante la comida y un mayor porcentaje de insectos que defecan durante los primeros 10,60 y 95 minutos después la alimentación. Aunque R. colombiensis presenta menor número de defecaciones que $R$. prolixus, es necesario tener en cuenta que $R$. colombiensis permanece mayor tiempo cerca del hospedero vertebrado, aumentando así la probabilidad de transmitir T. cruzial vertebrado.

De las 15 especies de Rhodnius descritas, tres de ellas se encuentran comúnmente en ambientes domésticos ( $R$. prolixus, $R$. pallescens y $R$. ecuadoriensis); las demás especies se registran principalmente en ambientes arbóreos como las brácteas de las palmas y las bromelias. Sin embargo, desde hace varias décadas, en varios países de América Latina se ha señalado el comportamiento de varias especies de Rhodnius que ingresan a las viviendas con frecuencias progresivas, entre las que se destacan $R$. neglectus, $R$. pictipes y $R$. nasutus (18). Más recientemente se presentaron evidencias sobre el papel putativo de $R$. robustus como vector extradomiciliario de la enfermedad de Chagas en el occidente de Venezuela, pues esta especie es atraída por la luz artificial, y la transmisión de $T$. cruzi podría ocurrir cuando se alimenta sobre las personas de las viviendas (19).

Hasta el momento no se conoce el papel de $R$. colombiensis en la transmisión de $T$. cruzien áreas en donde esta especie existe en cercanías al peridomicilio humano. Se ha comprobado que los adultos $R$. colombiensis invaden con frecuencia el domicilio humano, presentando elevadas prevalencias de $T$. cruzi, lo que aumentaría la 
probabilidad de transmisión al hombre y a los animales domésticos. De conformidad con lo anterior, es de gran importancia estudiar el posible papel de $R$. colombiensis en la transmisión de $T$. cruzi, especialmente en aquellas áreas en donde $R$. prolixus se ha controlado.

\section{Conflicto de intereses}

Los autores del presente artículo declaramos que no teníamos conflictos de intereses de orden académico, institucional u operacional en el momento de realización de la investigación.

\section{Financiación}

Este trabajo recibió financiación del Instituto Colombiano "Francisco José de Caldas" (Colciencias), proyecto 105-05-279-99, y del Fondo de Investigaciones de la Universidad del Tolima. También recibió apoyo internacional de la red ECLAT (European Community-Latin-American Network for Research on the Biology and Control of Triatominae).

\section{Referencias}

1. Galvão C, Carcavallo R, Silva RD, Jurberg J. A checklist of the current valid species of the subfamily Triatominae Jeannel, 1919 (Hemiptera, Reduviidae) and their geographical distribution, with nomenclatural and taxonomic notes. Zootaxa 2003;202:1-36.

2. Schofield CJ, Dujardin JP. Theories on the evolution of Rhodnius. Actual Biol 1999;21:183-97.

3. Moreno MJ, Galvão C, Jurberg J. Rhodnius colombiensis $s p$. $n$. da Colômbia com quadros comparativos entre estructuras fálicas do género Rhodnius Stal, 1859 (Hemiptera, Reduviidae, Triatominae). Entomol Vect 1999;6:601-17.

4. Zeledón R, Alvarado R, Jirón LF. Observations on the feeding and defecation patterns of three triatomine species (Hemiptera: Reduviidae). Acta Trop 1977;34:65-77.

5. Monroy E, Susunaga G. Caracterización biológica de las cepas de Trypanosoma cruzi aisladas de Rhodnius prolixus en el departamento del Tolima. (Tesis, biología). Ibagué: Universidad del Tolima; 1990. p.151.

6. Richa DS, Galvão C, Jurberg J. Biología do Rhodnius pictipes Stal, 1872 em Condições de Laboratório (Hemiptera, Reduviidae, Triatominae). Mem Inst Oswaldo Cruz 1994;89:265-70.

7. Soares RP, das Graças Evangelista L, Laranja LS, Diotaiuti L. Population dynamics and feeding behavior of Triatoma brasiliensis and Triatoma pseudomaculata, main vectors of Chagas disease in northeastern Brazil. Mem Inst Oswaldo Cruz 2000;95:151-5.

8. Martínez-Ibarra JA, Novelo López M, Hernández Robles MR, Grant Guillén Y. Influence of the blood meal source on the biology of Meccus picturatus Usinger 1939 (Hemiptera: Reduviidae:Triatominae) under laboratory conditions. Mem Inst Oswaldo Cruz 2003;98:227-32.

9. Wood SF. Importance of feeding and defecation times of insect vectors in transmission of Chagas' desease. $\mathrm{J}$ Econ Entomol 1951; 44: 52-54.

10. Dias E. Obsevações sôbre eliminação de dejeções e tempo de sucção em alguns triatomineos sulamericanos. Mem Inst Oswaldo Cruz 1956;54:11524.

11. Pipkin AC Sr. Domiciliary reduviid bugs and the epidemiology of Chagas' disease in Panama (Hemiptera: Reduviidae: Triatominae). J Med Entomol 1968;5:107-24.

12. Pippin WF. The biology and vector capability of Triatoma sanguisuga texana Usinger and Triatoma gerstaeckeri (Stal) compared with Rhodnius prolixus (Stal) (Hemiptera: Triatominae). J Med Entomol 1970;7:30-45.

13. Rocha DS, Fonseca AH, Costa FA, Jurberg J, Galvâo C. Desenvolvimento de Rhodnius pictipes Stal, 1872 alimentado através de membrana de silicone e em camundongos (Hemiptera, Reduviidae, Triatominae). Mem Inst Oswaldo Cruz 1997;92:553-8.

14. Buxton PA. The biology of a blood sucking bug Rhodnius prolixus. Trans Ent Sco Soc London 1930;78:227-36.

15. Friend WG, Choy CT, Cartwright E. The effect of nutrient intake on the development and the egg prodution of Rhodnius prolixus Stal (Hemiptera: Reduviidae). Can J Zool 1965;43:891-904.

16. Regis L. The role of the blood meal in egg-laying periodicity and fecundity in Triatoma infestans. Internat J Inver Reprod 1979;1:187-95.

17. Rocha DS, dos Santos CM, Cunha V, Jurberg J, Galvao C. Life cycle of Rhodnius brethesi Matta, 1919 (Hemiptera, Reduviidae, Triatominae), a potential vector of Chagas disease in the Amazon region. Mem Inst Oswaldo Cruz 2004;99:591-5.

18. Dujardin JP, García-Zapata MT, Jurberg J, Roelants P, Cardozo L, Panzera F, et al. Which species of Rhodnius is invading houses in Brazil?. Trans $\mathrm{R}$ Soc Trop Med Hyg 1991;85:679-80.

19. Feliciengeli MD, Dujardin JP, Bastrenta B, Mazzarri $\mathbf{M}$, Villegas J, Flores $\mathbf{M}$, et al. Is Rhodnius robustus (Hemiptera: Reduviidae) responsible for Chagas disease transmission in Western Venezuela? Trop Med Int Health 2002;7:280-7. 